\title{
Linx
}

Revue des linguistes de l'université Paris X Nanterre

$53 \mid 2005$

Le semi-figement

\section{Semi-figement et limites de la phrase figée}

Lexicon-Grammars and the limits of set expressions

\section{Antoinette Balibar-Mrabti}

\section{OpenEdition}

Journals

Édition électronique

URL : http://journals.openedition.org/linx/256

DOI : 10.4000/linx.256

ISSN : 2118-9692

\section{Éditeur}

Presses universitaires de Paris Nanterre

\section{Édition imprimée}

Date de publication : 1 décembre 2005

Pagination : $35-54$

ISSN : 0246-8743

\section{Référence électronique}

Antoinette Balibar-Mrabti, «Semi-figement et limites de la phrase figée », Linx [En ligne], 53 | 2005, mis en ligne le 11 février 2011, consulté le 19 avril 2019. URL : http://journals.openedition.org/linx/256 ; DOI : 10.4000/linx.256 


\title{
Semi-figement et limites de la phrase figée
}

\author{
Antoinette Balibar-Mrabti \\ Laboratoire MoDyCo (CNRS - UMR 7114)
}

\section{Remarques préliminaires de méthode : documentation et syntaxe}

La notion de «semi-figement» sera étudiée ici à partir d'une approche méthodologique désormais classique, celle des lexiques-grammaires. Je centrerai l'analyse sur un exemple, l'expression brûler ses vaisseaux. La méthode retenue place les propriétés morphologiques et syntaxiques dans une perspective d'application et de vérification systématiques sur le lexique, celui-ci étant traité aux dimensions des lexicographes. On sait que cette visée, novatrice dans les années 1970-1980, a conduit à un type de fusion entre dictionnaires et grammaires, les grammaires lexicalisées. Elle engage ici la pertinence des questions soulevées, leur visibilité, la nature des réexamens critiques. Je renvoie, dans $\operatorname{Linx} 53$, à la contribution de Christian Molinier qui apporte, à propos des constructions causatives, un exemple de résultats : une classification en taille réelle ${ }^{1}$; de même la contribution de Jean René Klein et Béatrice Lamiroy permet dans ce numéro une vue générale sur les lexiques-grammaires. Pour eux, le semifigement est à mettre au centre des leçons à tirer sur trente ans de programmes de recherches portant sur le figement.

\footnotetext{
1 Je renvoie également à la contribution ici-même de F. Dubois-Charlier et à son ouvrage Locutions en français (2004, en collaboration avec J. Dubois), complémentaire d'un Dictionnaire électronique des locutions de 5810 entrées (1995, chez les auteurs).
} 
L'expression brûler ses vaisseaux fait naturellement réfléchir sur la part de latin (comme de grec ancien) qui demeure inscrite, par traduction, dans les formes grammaticales et le lexique que nous appelons «ordinaires». Pour analyser cette transmission, j'examinerai l'apport en syntaxe d'une discipline externe: la métalexicographie. Celle-ci nous permet de faire l'histoire des dictionnaires qu'il est ici éclairant de compléter par l'histoire des manuels scolaires, grammaires et exercices, dont les auteurs se recoupent. A propos du Nonvean Larousse illustré étudié au $\$ 4$, j'ajoute en annexe documentaire (Annexe I) une grammaire de son directeur de publication, Claude Augé. L'apparentement des deux ouvrages est symptomatique des dispositifs d'acquisition collective, que notre modernité renforce. Pas d'exercices de langue sans utiliser les dictionnaires qui redoubleront, avec leurs variantes propres de présentation, les caractérisations syntaxiques et sémantiques, et contribueront aux routines d'apprentissage.

Au moment où la recherche documentaire prend de l'ampleur et gagne en efficacité, la constitution et les traitements de plus en plus élaborés et performants de gros corpus ${ }^{2}$ changent notre attitude devant les productions langagières. Mettre à l'épreuve nos intuitions d'acceptabilité, par des va-et-vient entre phrases et textes, reprend de l'importance en syntaxe à travers ces percées de la nouvelle documentation automatisée en linguistique. De fait, nous sommes renvoyés à l'exigence d'« attester » les données, donc portés à revisiter une bonne partie des approches de "grammaire et style » que la syntaxe avait reléguées du côté des descriptions dites «traditionnelles » ou encore «impressionnistes». Parmi les tâches qui mettent désormais en coordination étroite documentation et syntaxe, raffiner l'examen des sources sousjacentes à l'acquisition de la norme et de l'usage devient crucial. Traiter les manuels et les dictionnaires de la période 1850-1950 comme des documents, d'une valeur équivalente ou supérieure aux textes littéraires et journalistiques habituellement requis, me parait à l'ordre du jour. Ma question centrale sera celle-ci : comment optimiser la documentation?

\section{Brûler ses vaisseaux et la problématique de la phrase figée}

Dans les classifications de type lexiques-grammaires, la combinaison verbe-nom brûler ses vaisseaux (s'interdire de reculer) ${ }^{3}$, que les dictionnaires usuels et les manuels ${ }^{4}$ donnent à l'infinitif, constitue une séquence figée à condition d'en faire une phrase.

\footnotetext{
${ }^{2}$ Pour un tour d'horizon récent en linguistique des textes, je renvoie à l'étude de D. Malrieu, «La constitution de corpus : ses motivations, les méthodes, les questionnements possibles », Séminaire de l'Ecole doctorale "Connaissance, langage, modélisation» du 3 mars 2006, CNRS-Modyco, publications en ligne, edclm@u-paris10.fr.

${ }^{3}$ Ce sens sera étudié au $₫ 4$. Dans l’ensemble de cet article, annexes comprises, pour toute citation à partir de documents, on retrouvera en gras l'expression étudiée.

${ }^{4}$ On la trouve présente sur les cent ans du Petit Larousse illustré (1905-2005), dans les Grand Larousse, dans les Robert, dans le Littré; elle a deux possibilités d'entrée, comme unité polylexicale, l'entrée brûler et l'entrée vaisseaux. Pour des exemples de manuels, se reporter aux Documents annexes (I) déjà signalés.
} 
On part de la possibilité de construire le verbe et son complément dans un exemple comme :

(1) Léo (brûle + a brûlé) ses vaisseaux

où brûler est observable à un temps fini de la conjugaison dans une phrase minimale de même structure qu'une phrase libre comme :

(2) Léo lit un livre

On inclut obligatoirement l'argument sujet, indispensable à la formation du cadre phrastique d'analyse.

L'exemple (1) condense deux lectures grammaticales qui satisfont à la norme et à l'usage: il illustre un schéma valenciel; il coïncide avec des phrases simples déclaratives qui correspondent à un modèle canonique de grammaire et d'exercices usuels. Différentes possibilités de flexion verbale temporelle/aspectuelle sont ouvertes par ce cadrage phrastique minimal. Elles ont été formalisées dès les premiers projets de classification par tables (M. Gross 1968), en anticipation des études à mener complémentairement a posteriori sur corpus, que les systèmes actuels de traitement automatique des textes permettent d'explorer avec une précision croissante. Pour une synthèse récente sur les outils informatiques disponibles, je renvoie à Benoit Habert (2005) ; plus particulièrement ici, lorsque des lexiques-grammaires et des dictionnaires électroniques, autour des méthodes initiées par Maurice Gross ${ }^{5}$ en lexicologie et en syntaxe, sont à la base des modélisations informatiques, je renvoie aux recherches et aux résultats pionniers de Max Silberztein (1993) avec INTEX, puis NooJ ${ }^{6}$. Je renvoie également à Cédric Fairon (2004), à propos de la notion d'«incise », cruciale dans le repérage d'items (ou tokens) discontinus.

Dans l'exemplification, hors contexte, on respecte une tradition, celle de l'exemple forgé de dictionnaire, et on privilégie le présent et le passé composé de l'indicatif. La propriété définitionnelle utilisée est la structure NO VN1 commune à (1) et (2) ${ }^{7}$. L'indication du choix lexical constant dans (1) pour le deuxième argument N1 se note $C 1$ et sert à introduire la forme NOVC1 avec laquelle sont recensés 5848 emplois (C. Leclère 2002: 42) sur les 40000 formes figées de la classification. L'exemple d'école :

$$
\text { Léo a cassé sa pipe }
$$

en fait partie, de même que mettre les voiles, passer la main, etc. Pour la consultation des emplois, en version papier, sur le principe de la première classification présentée dans Maurice Gross (1975), on se donne les commodités d'un classement alphabétique, traditionnel en lexicographie. Les verbes (e. g. : brûler, casser, lire, mettre, passer) sont en entrées des classes, ou tables. Cela n'exclut pas de choisir en entrées les noms (e. g. : pipe, main, vaisseau $(x)$, voile(s)), de préférence aux verbes, dès lors que les unités considérées

\footnotetext{
${ }^{5}$ Pour la priorité donnée aux dictionnaires électroniques, en documentation et traduction assistées par ordinateur, voir M. Gross (1994).

${ }^{6}$ www.nooj4nlp.net.

${ }^{7}$ Une représentation élégante et fonctionnelle devenue classique.
} 
sont polylexicales. C'est la position du Lexis qui place brûler ses vaisseaux à l'entrée vaisseau. L'apport des dictionnaires électroniques est d'avoir rendu immédiates d'emploi et d'exécution ces virtualités de classement qui complexifiaient considérablement la reconnaissance et la production des expressions composées dans du texte.

Ces présentations lexicales, inscrites dans le terme «lexique-grammaire» luimême, sont subordonnées, en théorie, à la description d'ensemble, qui est d'abord syntaxique. Maurice Gross innove lorsqu'il parle délibérément dans son système, de phrase figée 8 . Il choisit la notion de «phrase figée » dans une perspective généralisante. Puisque toute expression figée se théorise dans une phrase, on est fondé à la désigner comme une phrase figée. En conséquence, sauf dans des cas peu nombreux, les phrases figées sont des mixtes de termes fixes et de termes dont la distribution est libre. Décrites par des structures de surface qui vont de pair avec leur identification automatique dans les textes, celles-ci se délimitent terme à terme dans une problématique d'ensemble, formulée dans Maurice Gross (1988) : quelles sont les limites de la phrase figée ? Cette problématique vaut en théorie pour toute partie du discours majeure. Elle inclut l'adjectif, l'adverbe, le nom, car le lexique-grammaire, on l'a dit, conserve cet héritage traditionnel, transmis dans nos habitudes orthographiques, avec sa dimension de dictionnaire d'emploi des mots dans les phrases.

\section{Sujet libre et flexion du déterminant dans C1}

Les exemples (1), (2), (3) ont tous un sujet libre restreint aux humains ${ }^{9}$ : une première réponse, immédiate, à la question des limites de la phrase figée. Les procédures de reconnaissance de leur zone fixe continue ou discontinue, permettant de retrouver les phrases dans du texte, ont deux versants, informatique et linguistique. Je reprendrai ici l'essentiel des caractérisations linguistiques que les modèles mettent en convergence avec les solutions informatiques associées.

Brûler ses vaisseaux se construit avec un déterminant possessif obligatoirement coréférent au sujet humain. On sait que cette particularité n'est pas exceptionnelle. Elle s'observe sur (3). Rappelons qu'elle entraîne une variation flexionnelle liée aux marques de personne du possessif en accord avec le choix du sujet de la phrase. Brûler entre ici dans la forme

$$
\text { (NO }=: N \text {-bum) brûler PossO vaisseaux }
$$

Les phrases :

$$
\text { Léo a cassé ta pipe }
$$

(6) Léo a brûlé vos vaisseaux

\footnotetext{
${ }^{8}$ Non sans jouer implicitement avec l'histoire du terme et ses différences de définition en grammaire contemporaine française et anglaise $:$ groupe $=$ phrase, phrase $=$ sentence.

${ }^{9}$ Voir les contributions de Dubois-Charlier (art. cit.), Klein et Lamiroy (art. cit.).
} 
qui violent la règle d'accord du possessif ne sont plus à considérer comme contenant la zone fixe qui déclenche l'analyse syntactico-sémantique de figement. Ce sont des phrases libres ordinaires. Cette propriété est partiellement masquée dans un dictionnaire traditionnel où le verbe est fixé dans une présentation à l'infinitif. A la forme du verbe répond alors la forme fixée à la troisième personne ses pour le déterminant coréférent au sujet laissé virtuel. Les procédures automatiques de flexion, initiées par Blandine Courtois et Max Silberztein (1990) avec le système de dictionnaires DELA, permettent la reconnaissance de la forme (4) avec un taux d'erreurs non nul mais réduit.

Collectées dans un listing, les combinaisons lexicales sont ensuite à analyser dans leur contexte, comme on reprend l'orthographe d'un énoncé à partir d'un correcteur d'orthographe qui localise, sans les corriger, des zones préférentielles et fournit ainsi des aides à la correction. La continuité des listings avec les tables de concordance de la tradition philologique la plus ancienne n'est plus à démontrer. De fait, ils sont appelés «concordanciers » ou "concordances » par les utilisateurs et les chercheurs. Réexaminer les implications et les continuités, en sciences du langage, de ces va-et-vient entre cadrages phrastiques minimaux et textes ${ }^{10}$ fait partie des questions les plus stratégiques de la recherche récente.

On sait que seul un contexte ${ }^{11}$, donné ou à construire, permettra ici de décider si l'expression, structurellement ambiguë pour (1) comme pour (3), prend son sens « ordinaire », calculable, ou prend un sens dont le calcul est plus détourné, donc à considérer comme "décalé », celui-ci allant précisément de pair avec les indices de figement. Un des artefacts de la phrase théorique, considérée en dehors d'un texte et d'un contexte, dont on se coupe par a priori de méthode, est de démultiplier les ambiguités $^{12}$. Pour les lever, les syntacticiens ont dû proposer des solutions de rechange aux commentaires habituels en grammaire et philologie. Maurice Gross $(1975$, op. cit.) invente les «commentaires de tables» dont il faut maintenant raffiner l'exploitation, compte tenu des acquis de l'automatique documentaire et linguistique dont il a été l'un des pionniers.

Les levées d'ambiguités instaurées et codifiées avec les premières grammaires structurales puis transformationnelles et génératives s'effectuent avec des faisceaux de tests devenus classiques. Par commutation, on a substitué ici à ses le déterminant vos dans (5), entraînant la rupture du sens figuré, et testé ainsi une première levée d'ambiguïté. On testera plus loin la substitution de bateaux à vaisseaux (\$5). Des propriétés transformationnelles sont mises en jeu. J'en donne maintenant des exemples (\$3) sans entrer dans les formalismes syntaxiques et sémantiques et leur historique. Ils sont abondamment décrits dans la littérature ${ }^{13}$. Ce qui m'intéressera ici, ce sont les phrases et plus généralement les énoncés en « récits» (\$3.2. et \$4) qui

\footnotetext{
10 Je renvoie sur ce point à G. Kleiber (2003), P. Le Goffic (2001-2005).

${ }^{11}$ C'est un problème classique des traducteurs repris en traduction automatique. Du français à l'anglais, voir M. Salkoff (1999).

12 Je renvoie sur ce point au petit ouvrage de C. Fuchs (1996); sur les rapports entre les ambiguités et les amphibologies traditionnelles, voir A. Balibar-Mrabti (juin 2007).

13 Voir par exemple le bilan-discussion d'I. Simatos (1997).
} 
permettent les jugements d'acceptabilité et le tri des sens par évaluation différentielle. Dans ce but, et de façon informelle, je parlerai de « reformulations ».

\section{Reformulations}

\subsection{Insertions, question, extraction, détachement}

Reprenons la forme définitionnelle NOVC1 et ses transformations associées. Celles-ci codifient les procédures de reformulation pour brûler ses vaisseau en vue d'évaluer ses degrés de blocage ${ }^{14}$. On réorganisera la partie fixe des phrases données $(\$ 1)$ :

(5) Léo (brûle + a brûlé) ses vaisseaux

Les opérations élémentaires classiques sont l'effacement, l’insertion, le réarrangement de l'ordre des items (les «token » en traitement automatique). Tous les tests considérés reposent sur la conservation du sens initialement choisi ou sa variation sans rupture. Il faudra donc éviter ici le «retour» au sens ordinaire (ou sens propre) et, chose plus délicate à réaliser et à mesurer, les jeux de langage tels qu'on les trouve en surabondance dès qu'on fait une investigation sur corpus. Ceux-ci sont largement des «bruits » pour le syntacticien. Ils nous placent en situation, certes, de discours, au sens le plus général, englobant la syntaxe, mais précisément hors des conditions d'observation théoriques qui favorisent l'analyse des propriétés strictement syntaxiques. Celles-ci demandent qu'on mette à distance les objectifs esthétiques et pratiques d'un texte extrait d'une œuvre littéraire ou d'un article de presse.

Pour faire apparaitre la spécificité des tests syntaxiques de reformulation et ce qui distingue une phrase théorique de la plupart des citations, l'exemple journalistique typique suivant (Documents annexes II) :

$$
\text { Cela s'appelle brûler ses vaisseaux...à petit feu }
$$

permettra un contraste éclairant. Le grammairien évitera le jeu de mot sur brûler et à petit feu parce que celui-ci parasite inutilement l'effort de définition et de répartition des différents sens en jeu, que l'on continue ou non à les appeler propres vs figurés. Amuser ou rire, avec une métaphore filée qui prolonge brûler par feu, n'appartient pas au noyau dur de la syntaxe et de la lexicologie. Ce qui ne veut pas dire qu'il n'existe pas des points de contact entre rhétorique et syntaxe et ils sont bien connus précisément avec la métaphore ou avec la métonymie. J'examinerai plus loin l'allusion historique (\$4) qui fait partie, dans la tradition lexicographique, de l'analyse des données qui ont fixé l'usage normé de brûler ses vaisseaux en français ordinaire contemporain.

Si on néglige une différence de registre (langue soignée/langue familière), brûler ses vaisseaux s'analyse à peu près comme l'exemple d'école casser sa pipe. Considérons le bloc d'exemples parallèles suivant :

14 Voir S. Mejri (dans ce numéro). 
Types d'insertions : adverbes, négation, phrases en incises

Il brûlera tôt ou tard ses vaisseaux / Il cassera tôt ou tard sa pipe

Il n'a pas brûlé ses vaisseaux / Il n'a pas cassé sa pipe

Il brûlera, je le crains, ses vaisseaux / Il a, parions-le, cassé sa pipe

Question, extraction, détachement

* Il a brûlé quoi? ses vaisseaux / * Il a cassé quoi? sa pipe

*? Ce sont ses vaisseaux qu'il a brûlés /* C'est sa pipe qu'il a cassée

*? Ses vaisseaux, il les a brûlés / *? Sa pipe, il l'a cassée

Ici la présence du possessif coréférent au sujet est un facteur supplémentaire de blocage donc, on l'a dit, de rupture possible avec le sens initial. On sait qu'elle interdit le test du passif et de la relativation dans des phrases libres ordinaires comme ${ }^{15}$ :

Ce roman policier a été relu par Luo

* Sa lettre a été relue par Luc

Ce roman policier que Luc a relu $<$ a été adapté au cinéma $>$

*Sa lettre que Luc a relue / *Sa lettre, que Luc a relue, $<m$ 'est destinée $>$

\subsection{Schémas de récits}

Pour clore cet aperçu classique des tests formels en jeu, j’ajouterai une remarque. Pour décider des restrictions d'emploi des formes fléchies du verbe, il ne suffit pas d'affirmer que brüler ses vaisseaux a une interprétation ponctuelle non durative $^{16}$ qui exclurait l'imparfait. Considérons les exemples suivants, reformulés à partir des définitions des dictionnaires Larousse (\$4.1):

(Cortés + Guillaume le Conquérant) a brûlé ses vaisseaux

(8) ?* (Cortés + Guillaume le Conquérant) brûlait ses vaisseaux

S'il est vrai qu'il peut exister une nuance d'acceptabilité au détriment du naturel de l'exemple (8) par rapport à (7), on voit que le choix du sujet, Cortés, Guillaume le Conquérant, repris d'interprétations historiques et littéraires traditionnelles, réalise les conditions d'un contexte dans un registre de langue soignée ${ }^{17}$, laissé implicite (ou

\footnotetext{
${ }^{15}$ Ici comme dans les reformulations précédentes l'étoile $\left(^{*}\right)$ indique une rupture du sens. Le possessif sa ne renvoie plus à $L u c$ donc la coréférence avec le sujet de relire disparait.

16 Sauf artifice langagier du type de l'exemple (6) donné plus haut qui force l'incompatibilité aspectuelle du verbe et de son modifieur. Pour un examen approfondi, je renvoie aux travaux de $\mathrm{M}$. Prandi à partir de Prandi (2002).

${ }^{17}$ Des contrastes sur le choix du sujet du verbe, comparables à celui qui nous intéresse entre (1) et (7), ont été analysés dès les premiers travaux en grammaire générative par J.-C. Chevalier (1976) chez $\mathrm{N}$. Ruwet. En voici des exemples à propos des verbes de sentiment et du sujet dit «agentif » dans un article de 1995 :

César méprisait Cassius.

! La Guerre du Péloponèse préoccupe Max.

Mes enfants me préoccupent.
} 
virtuel) en phrase simple théorique. Il suffit alors d'insérer la phrase dans un enchaînement ${ }^{18}$ conséquentiel :

(9) (Cortés + Guillaume le Conquérant) (débarque+ débarqua) avec sa flotte

Huit jours plus tard, il (brûle + brûlait) ses vaisseaux

dont le résultat est de lever le doute sur l'acceptabilité de (8). Autrement dit, l'exemplification en phrase simple est sous-tendue ici par un mini-récit conventionnel qui crée les conditions de son acceptabilité et avec celle-ci les conditions de l'emploi des temps du récit, imparfait et passé simple. Ce constat correspond à une interprétation connue des lexicographes et présente dans les manuels de rhétorique, l'allusion historique. Je dirai donc qu'une partie du travail contemporain visant à incorporer des figures dans la grammaire, parce qu'elles nous permettent de circonscrire des régularités d'interprétations sémantiques sur des sens décalés, différents des jeux de mots (\$1), doit désormais porter sur des figures de discours comme celle-ci.

Avec le développement des investigations automatiques sur corpus, il faut reprendre les figures de pensée et faire tout un travail de ré-appropriation de la citation. Comme observatoire de l'usage, les citations sont précieuses et même obligées. A une condition : le syntacticien garde toute liberté de manœuvre autour des reformulations. C'est dans cette perspective que je soumettrai ici une hypothèse de travail initiée par Jean-Paul Boons (1985) en termes de «schémas de récit» avant de présenter plus en détail l'allusion historique et ses potentialités. Dans une phrase simple de lexique-grammaire, prise hors contexte par artefact de méthode $(\$ 1)$, telle que :

\section{(10) Max a enfermé le lion dans la cage}

penser l'expression du lieu (e. g. : dans la cage) comme un «but» plutôt qu'une « origine », est indécidable, sauf si le cadrage phrastique va de pair avec un scénario qui permet d'assigner à la construction du verbe enfermer une interprétation aspectuelle résultative. Les scénarios enchaînent des événements selon différentes logiques. Dans cet exemple, Jean-Paul Boons signalait une impasse de raisonnement (son «effet Hamlet») : le lion est-il ou n'est-il pas dans la cage au moment où Max décide de l'enfermer ? Il proposait de lever l'ambiguité en recourant à l'arbitraire de « récits » qui fixeraient, en les énumérant, chacune de ces solutions alternatives dans les correspondances forme/sens.

Depuis les années 1985, l'analyse du sens s'est considérablement enrichie et déplacée sur des terrains spécifiques. Vingt ans plus tard, le terme "récit» n’apparaitt pas pour autant comme une étape dépassée de la réflexion d'ensemble sur les contreparties sémantiques des formes classées. Il reste porteur de recherches incontournables pour élucider et théoriser des types de micro-enchaînements syntaxiques qui relèvent de l'argumentation mais aussi des textes en s'inscrivant dans la tradition de la

\footnotetext{
18 La notion de «phrase double ( Hamon 2004) à propos de l'expression de la cause, offre ici des recoupements intéressants.
} 
narration-description et des topoi $i^{19}$. Pour contraindre l'interprétation de (10) avec le lieu comme but, on l'insérera dans un schéma de récit ${ }^{20}$ du type de :

(11) Le lion n'etait pas dans une cage. Max a enfermé le lion dans la cage.

Pour contraindre l'interprétation de (10) avec le lieu comme origine, on l'insérera dans un schéma de récit du type de :

(12) Le lion était dans une cage. Max a enfermé le lion dans la cage.

Ces solutions permettent de raisonner en termes de phrases simples observables sans passer par des calculs logico-sémantiques abstraits ni non plus par des énoncés phrastiques d'acceptabilité douteuse ou forcée.

\section{Emplois figurés et semi-figement}

\subsection{Réduire le sens décalé à un emploi métaphorique de verbe mais avec quel sens?}

La séquence verbale brûler ses vaisseaux est considérée par les dictionnaires comme une expression figurée. Ce type de solution ne se comprend pas en dehors d'une analyse approfondie des rapports entre rhétorique, grammaire et lexique. Je n'entrerai pas ici dans le détail de la discussion. Je renverrai à l'étude d'Isabel Gonzalez Rey (2002) et plus particulièrement ici à son chapitre 9 (Les constructions verbales figées: 167-243) dont je partage largement les analyses. Elle montre que les correspondances forme/sens se saisissent dans une tradition rhétorique qui ne cloisonne pas les figures de mots et les figures de pensée mais tout au contraire les conjoint. En conséquence, le sens décalé de brûler ses vaisseaux est interprétable comme un sens métaphorique (op. cit., p. 109), mais la métaphore, si métaphore il y a, est indissociable ici d'autres figures. Parmi elles, il convient de faire un sort particulier à l'allusion, explicite dans la tradition lexicographique elle-même. L'allusion fournit aux lexicographes les éléments d'interprétation qui permettent d'attribuer son sens décalé à brûler ses vaisseaux, dont le lien organique avec ses définitions les plus courtes, données plus loin dans le Lexis ou le Shorter Oxford English Dictionary, est patent. L'allusion apporte des récits conventionnels ordinaires ${ }^{21}$. Métaphore et allusion s'articulent. De façon exemplaire, il devient impossible de trancher entre figement et non figement. Le semi-figement est alors la seule réponse prudente à proposer.

\footnotetext{
19 Je renvoie sur ce point à C. Plantin (1998).

20 J'ai testé en termes de « conditions d'un même récit » des analyses d'adverbes de manière à double portée (Balibar-Mrabti 1987) pour des phrases comme Jean sourit malicieusement, et, par la même méthode, des levées d'ambiguïté entre adjectifs, épithètes ou attributs de l'objet, dans une phrase comme J'ai bu mon café froid (Balibar-Mrabti 1999), proches des énoncés préalables de J.-C. Anscombre (1996).

21 Que je pourrais qualifier de «livresques», pour donner un équivalent au terme anglais literature désignant toute source documentaire écrite dans le cadre d'une recherche quelconque, qu'elle soit littéraire, scientifique ou autre, sans hiérarchies culturelles.
} 
De fait, parler de «métaphore » sans couplage avec la figure de l'allusion n'a pas grand intérêt pour raffiner ici l'interprétation sémantique. On remarque l'impossibilité de définir l'emploi verbal considéré en termes de restrictions de sélection, une des bases de définition les plus opératoires en syntaxe pour trier et classer les emplois verbaux observés en phrase simple 22 . Des oppositions, classiques, de type concret/abstrait, humain/animal, etc., permettront de séparer éponger un déficit (métaphorique avec N1 abstrait) et éponger la table (non métaphorique avec N1 concret), couver une grippe (métaphorique avec N1 nom abstrait de maladie et sujet $N O$ humain) et couver des cufs (non métaphorique avec $N 1$ concret et sujet $N O$ animal). Elles seront inopérantes pour contraster brûler ses vaisseaux (métaphorique) et brûler des papiers (non métaphorique). Dans ces conditions, pourquoi réduire ici le sens décalé à une métaphore plutôt que d'en rester prudemment à la seule indication d'un sens figuré23 dans la tradition des lexicographes?

Les propriétés dites métaphoriques font partie avec les ambiguités (\$2) des artefacts de l'unité phrase en syntaxe. Comme figures de mots ou tropes, les métaphores sont avec les métonymies et les synecdoques une commodité terminologique pour renvoyer à des sens autonomisables, donc décrochables des unités textuelles de dimension supérieure à la phrase simple, lieu privilégié de l'observation des schémas valenciels. Dans les classifications des lexiques-grammaires, elles interviendront pour dégrouper des emplois verbaux et réduire les disjonctions homonymiques en proposant des traitements polysémiques.

Dans des conditions inter-langues, on sait que traduire permet de tester un invariant sémantique de référence sans qu'il soit nécessaire de produire un contenu de définition, ce qui règle la difficulté d'avoir à sortir des contraintes du cadre strictement phrastique pour mieux préciser les sens en jeu. On le vérifie aisément pour brûler ses vaisseaux qui se traduit directement, et de façon satisfaisante, aussi bien en espagnol ${ }^{24}$ qu'en anglais. Ces possibilités de traduction, suggérées par les sujets Cortés, Guillaume le Conquérant, donnés dans les exemples (7)-(9), jouent alors directement dans la décision de ranger l'expression parmi les emplois figurés assimilés à des métaphores parce que le sens en jeu est décroché d'un sens «propre». On raisonnera en termes de moules syntaxiques communs, entre langues modernes, une terminologie proche de la notion traditionnelle en philologie de «traduction littérale » ou de «calque syntaxique ». De ce point de vue, les observations de grammaire comparée sont particulièrement fructueuses et exploitées (Klein et Lamiroy, art. cité).

En conclusion, j'attirerai l'attention sur le point suivant. On remarque qu'il serait difficile, à l'intérieur du français, de trouver un verbe simple synonyme de brûler

\footnotetext{
22 Sur le principe des tables de M. Gross, voir particulièrement M. Salkoff (1983) pour l'anglais.

23 C'est le choix terminologique du dictionnaire électronique de J. Dubois et F. Dubois-Charlier Les Verbes français (1994-1997). Pour une analyse réactualisée de ce dictionnaire je renvoie à Langue française 153 (mars 2007), J. François, D. Leeman, D. Le Pesant, éds.

24 Traduit par quemar los naves et signalé dans tous les dictionnaires espagnols à propos de la conquête du Mexique par Cortés. Comme en français, ou en anglais, l'allusion historique fonctionne parallèlement à l'allusion biblique ou littéraire (J.-C. Anscombre, communication personnelle), ce qui rend intéressante l'étude des trois traditions, français-espagnol-anglais, en méta-lexicographie et en didactique comparées.
} 
ses vaisseaux, à la façon dont on fait correspondre deux à deux, en négligeant le changement de registre, les exemples d'école casser sa pipe = mourir; quand les poules auront des dents $=$ jamais. Il existe cependant une nuance intéressante: c'est l'approximation brüler ses vaisseaux $=$ couper les ponts. Reportons-nous au Robert \& Collins senior, français/anglais anglais/français (2001). Il nous donne l'équivalence brûler ses vaisseaux $=$ to burn one's boats $=$ to burn one's bridges qui met en évidence une commutation de l'anglais entre boats et bridges. Transposée en français, elle conduirait à relier vaisseaux et ponts. Le dictionnaire ne définit pas l'invariant sémantique de référence. Il lui suffit de traduire pour réaliser ses objectifs d'outil de travail bilingue. Rapprochons deux définitions de dictionnaires monolingues :

fig., to cut oneself off from all possibility of retreat

(The Shorter Oxford English Dico, 1973, Clarendon Press Oxford)

se couper la retraite, accomplir un acte qui ne permet plus de reculer

(Dictionnaire de langue française, Lexis, 1979, Editions Larousse)

Le rapprochement montre une parenté directe de formulation, avec les trois parallélismes se couper/to cut oneself off, retraite/ retreat, permettre/possibility. Dans la tradition des définitions intuitives par mots synonymes, couper définissant brüler serait-il un bon candidat pour réaliser une commutation sur le verbe, dans l'expression française ? Pas exactement, puisqu'on ne dit pas * couper ses vaisseaux et qu'il faut prendre en bloc couper les ponts. Paradoxalement, l'anglais ne dispose $\mathrm{pas}^{25}$ de cette expression intermédiaire, susceptible d'être réinterprétée ${ }^{26}$ ici comme étant approximativement équivalente (\$5).

Ces rapprochements servent à souligner l'importance des approches de lexique et grammaire comparés dans l'étude des invariants sémantiques ${ }^{27}$ de référence. Ces invariants se fixent en partie à travers des approximations des traducteurs, en synchronie comme en diachronie (\$4.2.). Une des tâches de la linguistique, avec les nouvelles possibilités de recherches documentaires, est de raffiner notre connaissance des étapes de formation des relations formes/sens. Si nous sommes dans un cadre méthodologique de lexiques-grammaires, il s'agit de mieux penser la place des informations historiques et didactiques, de faire entrer de façon cohérente des données d'observation, disponibles en termes de monographies lexicologiques ${ }^{28}$. Par conséquent je m'intéresserai maintenant à l'interprétation figurée de brûler ses vaisseaux qui explique le plus directement, à partir d'une recherche documentaire sur les premiers dictionnaires Larousse, le contenu de définition proposé pour cette expression, ni tout à fait calculable ni tout à fait opaque.

\footnotetext{
${ }^{25}$ Le Robert \& Collins senior la traduit par to break off communications with somebody.

${ }^{26}$ Les ré-analyses d'expressions figées sont un problème classique qui ne sera pas approfondi ici.

${ }^{27}$ Pour une définition en termes de transducteurs, je renvoie à J. Labelle (1995). La discussion peut être élargie avec la notion de "passe-partout translation » utilisée par M. Salkoff (1999, op. cit.).

28 Un très bon exemple de monographies lexicologiques : danser la sarabande (Rey, 1977 : 226-236).
} 


\subsection{La figure de l'allusion historique : mini-récits et commentaires abrégés}

S’il y a bien un sens décalé, repérable et transposable par une traduction littérale dans un groupe de langues, dont font partie l'anglais, l'espagnol ou encore l'allemand, mais non l'italien (\$4.1), quel serait ce sens, différent d'une métaphore comme figure de mot, qui resterait distinct d'un sens totalement bloqué, et comment le définir en tenant compte de la norme et de l'usage ? L'histoire des dictionnaires nous donne précisément des sources en français. Elle nous offre des moyens d'observer dans quelles conditions textuelles s'établit la relation forme/sens qui nous intéresse ici. Pour cela, il faut réinterpréter la problématique initiale de la phrase figée (\$1) et dire: comment délimiter la phrase figée dans du texte ?

Considérons dans le Nouveau Larousse illustré (1896-1904, Claude Augé, dir.), la présentation suivante (deuxième tome, p. 310):

Brûler ses vaisseaux. Sens figuré. Voir la rubrique allusion historique [...]

Allusion à la conduite d'Agathocle et de plusieurs grands capitaines, que l'histoire nous représente incendiant les vaisseaux qui les avaient portés sur les bords ennemis, afin que leurs soldats, privés de toute espérance de retraite, fussent déterminés à vaincre ou à mourir. (Ces mots, qui ont passé en proverbe, signifient que par une initiative hardie, l'on se met dans l'impossibilité de reculer) [c'est moi qui souligne].

Il est intéressant de rechercher dans le même dictionnaire ce qu'il est dit d'Agathocle (premier tome, p. 114) car on y trouve précisément brûler ses vaisseaux dans le sens à partir duquel le sens figuré est déduit, autrement dit dans un type de sens propre de référence :

\section{Agathocle}

Tyran de Syracuse [...] Assiégé dans Syracuse en 311, il quitte cette ville, débarque en Afrique, brûle ses vaisseaux et marche sur Carthage en soumettant toutes les villes du littoral. Mais dans une seconde campagne (310), il est battu [... ]

Le récit historique éclaire l'allusion qui résumait les faits et les commentait à travers une leçon à tirer, qui leur confère une valeur exemplaire, qualifiée de « proverbe ». Une raison traditionnelle supplémentaire pour justifier le sens métaphorique: brûler ses vaisseaux, c'est se conduire comme... Mais s'il y a métaphore, c'est par le raccourci d'une comparaison, développée dans un parallélisme. Je reprends maintenant mon exemple initial :

$$
\text { Léo (brûle }+ \text { a brûlé) ses vaisseaux }
$$

Je remarque qu'il est en conformité avec la tradition rhétorique et juridique de l'exemple qu'Alain Rey (1995) fait relever des trois concepts de paradigma (ce qui est montré), d'exemplum (ce qui est extrait), de citatio (ce qui est appelé, invoqué). Je leur ajoute : ce qui est traduit.

Incendiant ${ }^{29}<$ les vaisseaux...> est un synonyme de brûler employé dans son sens propre et un calque lexical du latin d'origine incendi ${ }^{30}$. Il n'est pas possible ici de

\footnotetext{
${ }^{29}$ Souligné par moi dans le texte (Allusion, Nonveau Larousse illustrè).
} 
détailler la comparaison des quatre documents que j'ai rapprochés : le texte latin et ses adaptations françaises sous trois formes, la traduction pour spécialistes de Marie-Pierre Arnaud-Lindet (2003), l'«allusion» du Larousse (1896-1904), le « récit historique » de référence donné à l'entrée Agathocle. Ce dernier se détache des autres documents par la modernité du lexique et des enchaînements phrastiques, effectués sur le principe de la phrase simple, coordonnée, au présent de l'indicatif. Je me limiterai à faire remarquer qu'on a déjà ici le style des «schémas de récit» ou "mini-récits » contemporains qui nous intéressent (\$3.2).

Un regard rapide sur des dictionnaires postérieurs montre qu'ils proposent la même analyse d'ensemble dans une démarche générale qui abrège de plus en plus l'explication initiale. L'«allusion historique» va de pair avec un exercice oratoire d'amplification, désormais vieilli. Le plus intéressant est d'observer que les futures définitions lexicographique abrégées sont déjà contenues dans la formule ${ }^{31}$ résumée entre parenthèses, et traitée en proverbe, qui en est leur modèle direct dans le Nouveau Larousse illustré. Car cette formulation est déjà en conformité avec les définitions du Lexis ou du Shorter Oxford English Dictionary (\$4.1) qui se sont alignées sur la simplification qu'elle amorçait, avec des retouches de vocabulaire et de syntaxe qui ont permis d'actualiser les formulations antérieures, elles-mêmes partiellement vieillies.

La narration, et bien davantage encore la description, seraient-elles de plus en plus évincées par l'apport documentaire de l'image ${ }^{32}$ dans les démarches lexicographiques et encyclopédiques contemporaines ? La question est difficile. La forme « récit» reste éminemment moderne quand elle tend vers des schémas de phrases simples enchaînées par parataxe. Avec l'entrée Agathocle, analysée à sa juste place, dans la série historique de référence des dictionnaires Larousse, elle nous offre ici une source culturelle directement exploitable : c'est le «sens propre » de référence de la figure. Le "sens figuré », ancré culturellement dans la forme, très composite en rhétorique, de l'«allusion », est lui-même typé de façon éclairante en lexicographie du 19 ème siècle. En relation avec les exercices d'acquisition qui lui sont associés et qui contribuent à fonder des modes de penser et de raisonner par «stéréotypes » ou "clichés », il s'expose sur un mode adapté à la formulation de son sens second par rapport au sens propre: l'«explication» scolaire. De façon atomisée, celle-ci fonctionne sur le mode des réponses à apporter aux questions posées, du type: «Expliquez les locutions ou proverbes suivants...», «Dire le sens de chacune des locutions suivantes en appuyant l'explication d'un exemple» (Documents annexes I), dont le dictionnaire donne les solutions. Nous avons oublié l'exercice rhétorique d'amplification. Les exercices scolaires courants sont maintenant les «commentaires de textes » et leurs « résumés ». La forme « récit» apporte le sens propre de référence. Le « résumé de commentaire » fournit le sens décalé contemporain.

30 A partir de l'écrivain latin Justin qui apporte le mythe initial du stéréotype. Voir Documents annexes (III).

31 Se reporter aux mots que j'ai soulignés.

32 Le fonctionnement de la narration et de la description est à comprendre en couplage avec l'image qui, à cette époque, est une gravure. Une des sources de nos clichés d'expression collective, verbale et visuelle, sont à l'œuvre et se visualisent dans ce dictionnaire et les premiers Larousse. Je renvoie sur ce point à H. Meschonnic (1991). 


\section{Segment figé et texte}

Le cadre théorique retenu, morphologique et syntaxique, permet une description formelle de brûler ses vaisseaux, centrée sur le traitement des propriétés de flexion et de combinaison des mots, en «surface » à l'écrit. Dès les premières hypothèses de description, il est défini par Maurice Gross en vue des traitements automatiques (\$1). Il conduit naturellement à s'interroger sur l'utilisation effective d'une telle expression dans du texte contemporain imprimé ordinaire. Ici, la procédure est d'autant plus simple que nous pouvons prendre directement cette séquence à l'infinitif et négliger ses variations $(\$ 2)$ pour obtenir des résultats déjà intéressants. Nous vérifions immédiatement des propriétés classiques, dont la base de définition est cadrée, dans les lexiques-grammaires, à partir des constructions complétives (dès M. Gross 1975, op. cit.). En français contemporain, celles-ci fondent les parallélismes entre infinitif et temps finis (indicatif et subjonctif). Les textes traités, tout particulièrement ceux de la presse écrite, nous montrent que l'infinitif s'introduit de cette manière, en position d'argument phrastique ${ }^{33}$.

Je commenterai en conclusion l'emploi attesté ${ }^{34}$ en 2003 de la variante brûler ses bateaux. On trouve chez Amélie Nothomb dans son roman Antécbrista (Paris, Albin Michel 2003 ; Le livre de poche 2005, p. 132) le paragraphe suivant :

J'étais sidérée de voir combien elle s'y prenait mal. Ignorait-elle donc qu'elle avait la partie facile ? Elle s'enferrait dans l'agressivité qui était la stratégie la plus bête ; mon père avait pour elle un tel attachement qu'elle eût pu alléguer les motifs les plus invraisemblables, il eût marché. Au lieu de quoi, elle brûlait ses bateaux en pure perte.

Ce paragraphe nous montre, par vérification en contexte, que brûler ses bateaux s'interprète dans les sens discutés au $₫ 4$, incluant l'approximation avec couper les ponts. Nous constatons par cette attestation qu'il est parfaitement possible de substituer au terme vaissean le nom synonyme bateau. A condition de garder le pluriel et la coréférence obligatoire du déterminant, le sens décalé spécifique se maintient. Nous avons la preuve, par consultation du paragraphe dans son ensemble, que la combinaison verbe-nom réalisée s'effectue sans introduire de jeu stylistique, notamment satirique, susceptible de parasiter l'interprétation.

Cela aurait pu faire partie des commutations ( $\$ 2$ et $\$ 4.1)$ étudiées. La substitution de termes synonymes est l'un des tests habituellement pratiqués pour évaluer les degrés de figement. Lorsqu'il met en équivalence les noms nord et boussole, l'exemple d'école perdre le nord a cette propriété. De plus, comme Jean-Claude Anscombre l'a montré à partir des proverbes, la modernisation du lexique n'a rien de surprenant à l'intérieur d'un moule bien défini donc systémique. Remplacer vaisseau, vieilli en dehors de ses emplois techniques, tels que vaisseau sanguin, vaissean spatial, capitaine/ lieutenant/enseigne de vaissean (Petit Larousse 2005), fait donc partie des variations

\footnotetext{
33 Pour le détail des observations, se reporter aux Documents annexes (II).

34 Que la recherche donnée en Documents annexes (II) n'avait pas détecté mais qu'on peut repérer sur Internet.
} 
typiques d'un semi-figement. Mais les classifications (Dubois-Charlier, art. cit. ; Klein et Lamiroy, art. cit.) mettent bien en évidence qu'elles n'en sont pas prédictibles pour autant de façon simple.

Il revient ici à un texte de fiction, qu'il nous fasse ou non l'impression d'être bien ou mal écrit - là n'est pas la question -, de créer les conditions qui nous rendent acceptable et naturelle cette variation lexicale qu'à défaut d'attestation de ce genre nous n'aurions pu présenter ici que sous l'apparence d'une pure spéculation. Les problèmes du semi-figement débouchent sur la question particulièrement délicate à trancher de la vraisemblance des phrases que nous voudrions classer comme ordinaires sans jamais tomber dans l'arbitraire dérangeant des exemples forcés.

\section{DOCUMENTS ANNEXES}

\section{Annexe I}

\section{Acquisition de la grammaire et du style dans les manuels de français}

1) Un petit livre d'exercices de grammaire pour le Cours Moyen, réalisé par Claude Augé, continuateur de Pierre Larousse, choisit, au chapitre «Eléments du style », l'exemple Brûler ses vaisseaux pour définir dans la leçon sur "proverbes et locutions » la notion de «locution proverbiale» (C. Augé, Grammaire, Livre de l'élève, Librairie Larousse, sans date, p. 257, fonds documentaire R. Balibar) :

\section{Proverbes.- locutions.}

[...] Certaines locutions, sans présenter un sens complet comme les proverbes, offrent des images si justes ou si pittoresques que l'usage les a consacrées et en a fait des expressions que l'on ne peut modifier. On les appelle locutions proverbiales. Ex : Brûler ses vaisseaux, c'est s'engager dans une affaire de telle sorte qu'on ne puisse plus reculer.

Exercices. - Expliquez les locutions ou proverbes suivants : [...] Etre comme l'oiseau sur la branche. Tomber de Charybde en Scylla.

2) Plus près de nous, Henri Bonnard, dans ses Exercices de style, de versification et d'histoire de la langue française (1965, en collaboration avec R. Arveillé, Paris, SUDEL) qui accompagne sa Grammaire française (1950-1971, Paris, SUDEL) propose le même genre d'exercices scolaires au chapitre Stylistique des mots (p. 45):

Dire le sens de chacune des locutions suivantes en appuyant l'explication d'un exemple :

Coucher à la belle étoile/Etre pris entre deux feux/Brûler ses vaisseaux

Dans la tradition de tels exercices, qui contribue avec les dictionnaires à fixer donc à fonder l'usage, une expression comme celle-ci sera rapportée à un invariant sémantique de référence et jugée «stylistiquement enrichie ». C'est ce que fait Maurice Gross (1995) dans sa grammaires locale de l'expression des sentiments modélisée dans des graphes ou automates finis en vue d'une reconnaissance automatique sur corpus. 


\section{Annexe II \\ Consultation de gros corpus ${ }^{35}$}

- Presse écrite : vie politique

Prise à l'infinitif, la séquence brûler ses vaisseaux donne immédiatement des résultats. On la trouve dans la presse écrite (Base Europresse www.bpe.europresse.com.). Voici comment nous l'observons dans deux articles récents :

L'Express 13 septembre 2004, Fabius un pied dans le Rubicon

Cela s'appelle brûler ses vaisseaux ... à petit feu. En lieu et place d'une réponse claire et précise $[\ldots]$

Les Echos 4 février 2004, OPA,OPE, épopée

Et on attendra le dernier chant pour savoir si l'attaquant a eu raison de brûler ses vaisseaux

Dressons maintenant la liste des emplois qui introduisent l'expression à l'infinitif. L'expression occupe la position d'un argument (phrastique). En voici des exemples :

\section{(A)}

qui se condamne à......

a eu raison de

hésitera à ....

aurait conseillé au candidat de ....

Semble prêt à ....

tout en se désolant de devoir...

L'expression apparait dans une phrase subordonnée à l'infinitif :

(B)

A condition de ne pas brûler ses vaisseaux

donc dans des phrases qui s'analyseront comme phrases complexes avec enchâssement (type (A)), avec des connecteurs (type (B)). On remarquera également l'interrogation :

(C)

A quoi bon brûler ses vaisseaux à l'avance ?

ramenable au type (A) par ellipse d'un verbe de demande. Les journaux conservent à l'expression sa filiation gréco-latine d'inspiration politico-militaire à partir de Diodore et Justin.

- sources FRANTEXT, GALLICA : prose littéraire et thématique psychologique et sociale

Les sources donnent des auteurs comme Mme de Staël, Balzac, George Sand, les Goncourt. Un exemple :

\footnotetext{
35 Je voudrais remercier Brigitte Drouhin, documentaliste à la Bibliothèque universitaire de l'Université de Picardie Jules Verne, pour cette recherche approfondie dont je ne donne ici que quelques extraits.
} 
Cette grande et belle personne assez hardie pour brûler ses vaisseaux, comme Alexandre, au début de la vie, et commencer la lutte par une faute mensongère, disparut du théâtre après y avoir introduit un violent élément d'intérêt.

(Balzac La vieille fille, 1837)

- Internet : politique et psychologie sociale

Voici un site récent Brûler ses vaisseaux (http://vacarme.eu.org/areticle229.html) consacré aux grévistes de la faim. Il est intéressant pour la synthèse qu'il représente des thématiques énumérées.

[...] Il faut bien voir ce qui fait «tenir » une grève, c'est d'abord sa structure tactique : entamer une grève de la faim, c'est précisément brûler ses vaisseaux - on sort de la clandestinité, et on se lie soi-même à une menace faite à l'adversaire, menace qui n’a de chance d'être prise au sérieux que si on est réellement lié [...]

(entretien avec Johanna Siméant , 2002)

La solidité du stéréotype est telle qu'on le trouve à l'identique, répertorié dès le Grand Dictionnaire universel (1865-1876) de Pierre Larousse, avec une phrase comme celle-ci, attribuée à Marat :

$<$ le roi mort il n'y aura plus moyen de reculer> il faut maintenant brûler le vaisseau <qui nous a conduit $>$, afin que, n'ayant plus ni salut à attendre $[\ldots]$, ni merci à glaner $[\ldots]$, nous combattions $[. .$.$] pour maintenir la République.$

Au 18 ème siècle le pluriel n'est pas obligatoire pour vaisseau et la relative qui nous a conduit permet d'assurer la coréférence sans que le déterminant possessif (notre) soit requis.

\section{Annexe III}

\section{Source latine des documents lexicographiques étudiés}

Justin (Abrégé des Histoires Philippiques, Livre 22, 6.4.) :

Sic consolatis militibus uniuersas naues consentiente exercitu incendi jubet, ut omnes scirent auxilio fugae adempto aut uicendum aut moriendum esse / Ayant ainsi réconforté les soldats, il ordonne avec l'accord de l'armée de brûler tous les navires afin que tous sachent qu'il fallait vaincre ou mourir, le recours à la fuite ayant été ôté.

(texte établi et traduit par Marie-Pierre Arnaud-Lindet, 2003, C) Corpus scriptorum latinorum, a digital library of Latin literature) 


\section{RÉFÉRENCES}

Anscombre, J.-C. (1996), « Partitif et localisation temporelle», Langue française 109, L. Kupferman, éd., La préposition 'de'.

Anscombre, J.-C. (2007), (dans ce numéro), «Les proverbes: un figement du deuxième type ?»

Balibar-Mrabti, A. (1987), "Règles formelles et règles rhétoriques sur un cas d'analyse d'adverbes », Lingristicae Investigationes, IV : 1.

Balibar-MrabTi, A. (1999), «Les adjectifs chaud et froid comme attributs de l'objet », Langages 133, E. Laporte, éd., Lexique-grammaire des adjectifs.

Balibar-Mrabti, A. (2002), "Exemples lexicographiques et métaphores », Langue Française 134, A. Balibar-Mrabti et M. Conenna, éds., Nouvelles approches de la Métaphore.

BALIBAR-MraBti, A. (2004), «Lexique-grammaire et extensions lexicales : note sur le semifigement », Lingvisticae Investigationes Supplementa 24, Amsterdam/Philadelphia, Benjamins.

Balibar-Mrabti, A. (2007), «Phrases simples et exemplification dans Les Verbes français. Une réflexion sur on comme sujet », Langue française 153.

BoOns, J.-P. (1985), «Préliminaires à la classification des verbes locatifs : les compléments de lieu, leurs critères, leurs valeurs aspectuelles ", Lingvisticae Investigationes, IX : 2.

Chevalier, J.-C. (1976), «Le jeu des exemples dans la théorie grammaticale. Etude historique ", in J.-C. Chevalier et. al., éds, Grammaire transformationnelle : syntaxe et lexique, Presses universitaires de Lille.

Compere, M.-M. et Chervel, A. (1997), éds, Les humanités classiques, Paris, INRP.

Courtois, B. et SilberzTein, M. éds. (1990), Langue française 87, Dictionnaires électroniques du Français.

Dubois-Charlier, F. (2007) (dans ce numéro). «A propos de certaines locutions en français ».

Dubois, J. et Dubois-Charlier, F. (1994-1997), Les Verbes français, Paris, Larousse-Bordas.

Dubois, J. et Dubois-Charlier, F. (2004), Locutions en français, Aix en Provence, chez les auteurs.

FAIRON, C. (2004), «Une étude de corpus pour éclairer la question du verbe de l'incise en français », Lingvisticae Investigationes Supplementa 24, Amsterdam/Philadelphia, Benjamins.

Francois, J., Leeman, D., Le Pesant D., éds., (2007 juin). Langue française 153, Autour du dictionnaire des verbes de J. Dubois et F. Dubois-Charlier.

FuCHS, C. (1996), Les ambiguités du français, Gap/Paris, Ophrys.

GonZalez Rey, I. (2002), La phraséologie du français, Toulouse, Presses Universitaires du Mirail.

Gross, M. (1968), Grammaire transformationnelle du français. Syntaxe du verbe, Paris, Larousse. 
Gross, M. (1975), Méthodes en syntaxe, régime des constructions complétives, Paris, Hermann.

Gross, M. (1988), «Les limites de la phrase figée », Langages 90, L. Danlos, éd., Les locutions figées.

Gross, M. (1994), "Dictionnaires électroniques et traduction automatique », Langages 128, J.-R. Ladmiral, éd., Le traducteur et l'ordinateur.

Gross, M. (1995), « Une grammaire locale de l'expression des sentiments », Langue française 105, A. Balibar-Mrabti, éd., Grammaire des sentiments.

HABERT, B. (2005), Instruments et ressources électroniques pour le français, Gap/Paris, Ophrys :.

Hamon, S. (2004), "Pourquoi la cause est-elle essentiellement prédicative?», Revue de Sémantique et Pragmatique, numéro 15/16.

KLEIBER, G. (2003), « Faut-il dire Adieu à la phrase ? », L'Information grammaticale 98.

KLEIN, J.-R. et LAmiroy, B. (2007), (dans ce numéro), « Le problème central du figement est le semi-figement».

LABELLE, J. (1995), «Lexique-grammaire et variation en français », in J. Labelle et C. Leclère, éds., Lexiques-grammaires comparés en français, Actes du colloque international de Montréal (3-5 juin 1992), Amsterdam/Philadelphia, Benjamins.

LECLERE, C. (2002), "Organization of the lexicon-grammar of French verbs », Lingvisticae Investigationes, $\mathrm{XXV}, 1$.

LEEMAN, D. (1998), « La métaphore dans la description des prépositions », Verbum, XX, 4.

LE GOFFIC, P. (2001-2005), «La phrase revisitée », Le Français Aujourd’hui 148.

MejRI, S. (2007), (dans ce numéro), «Figement absolu ou relatif : la notion de "degrés de figement"».

MesChONNIC, H. (1991), Des mots et des mondes, dictionnaires, encyclopédies, grammaires, nomenclatures, Paris, Hatier.

MOlinieR, C. (2006), (dans ce numéro), «Sur les constructions causatives figées du français ».

Plantin, C. (1998), "Les raisons des émotions », in M. Bondi, éd., Forms of argumentative discourse/ Per un'analisi linguistica dell'argomentare, Bologne, CLUEB.

Prandi, M. (2002), "La métaphore: de la définition à la typologie », Langue française 134, A. Balibar-Mrabti et M. Conenna, éds., Nouvelles approches de la métaphore.

Rey, A. (1977), Le lexique : images et modèles, du dictionnaire à la lexicologie, Paris, Armand Colin.

REY, A. (1995), «Du discours au discours de l'usage : pour une problématique de l'exemple», Langue française 106, Alise Lehmann, éd., L'exemple dans le dictionnaire de langue.

Ruwet, N. (1995), «Les verbes de sentiment peuvent-ils être agentifs ? », Langue française 105, A. Balibar-Mrabti, éd., Grammaire des sentiments.

SALKOFF, M. (1983), «Bees are swarming in the garden, a systematic synchronic study of productivity », Language 59, 2. 
Antoinette Balibar-Mrabti

SAlKoff, M. (1999), A French/English Grammar. A Contrastive grammar on translational principles, Lingvisticae Investigationes Supplementa 22, Amsterdam/Philadelphia, Benjamins.

SILBERZTEIN M. (1993), Dictionnaires électroniques et analyse automatique de textes. Le système INTEX, Paris, Masson.

Simatos, I. (1997), «Expression idiomatique, expression figée, prédicat complexe », in B. Fradin et J. M. Marandin, éds., Mots et grammaires, Paris, Didier Erudition. 